\title{
Toward demonstrating controlled-X operation based on continuous variable four-partite cluster state and quantum teleporters
}

\author{
Yu Wang, Xiaolong Su $*$ Heng Shen, Aihong Tan, Changde Xie, and Kunchi Peng \\ State Key Laboratory of Quantum Optics and Quantum Optics Devices, \\ Institute of Opto-Electronics, Shanxi University, Taiyuan, 030006, People's Republic of China
}

\begin{abstract}
One-way quantum computation based on measurement and multipartite cluster entanglement offers the ability to perform a variety of unitary operations only through different choices of measurement bases. Here we present an experimental study toward demonstrating the controlled-X operation, a two-mode gate, in which continuous variable $(\mathrm{CV})$ four-partite cluster states of optical modes are utilized. Two quantum teleportation elements are used for achieving the gate operation of the quantum state transformation from input target and control states to output states. By means of the optical cluster state prepared off-line, the homodyne detection and electronic feeding forward, the information carried by the input control state is transformed to the output target state. The presented scheme of the controlled-X operation based on teleportation can be implemented nonlocally and deterministically. The distortion of the quantum information resulting from the imperfect cluster entanglement is estimated with the fidelity.

PACS numbers: 03.67.Lx, 42.50.Dv, 42.50.-p
\end{abstract}

\section{INTRODUCTION}

Developing faster and faster computers is a long process. Quantum computers (QCs) based on the fundamental principles of quantum physics, such as coherent superposition and entanglement of quantum states, promise super-fast and powerful computation capacities for the future over present classical computers. In recent years, esoteric and attractive ideas about quantum computers were being converted into visible realization step by step along with experimental demonstrations of various quantum logic operations in both discrete variable (DV) and continuous variable (CV) domains [1-8]. Different models for quantum computation, typically the conventional circuit model and the so-called cluster model, were proposed and experimentally realized [9-18]. According to the cluster-state model initially proposed by Raussendorf and Briegel [15],the actual computation can be completed only by a sequence of single-qubit projective measurements with classical feed-forward of the measured results with the help of the special multiparticle entanglement between individual subsystems of the cluster state prepared off-line. Due to the irreversibility of measurements the cluster-based QC is also inherently time-irreversible, and thus is named the one-way QC. The most important feature of cluster QCs is its universality, i.e. any quantum circuit can be implemented on a suitable cluster state [15]. Exploiting four-photon cluster states, the performances of one-way DV QC were experimentally demonstrated [16-18].

In 1999, Lloyd and Braunstein extended the quantum computation to the $\mathrm{CV}$ region and derived the necessary and sufficient conditions for achieving universal CV QC [19]. Successively, the CV QC with optical coherent states [20] and the encoding schemes for CV computation were proposed [21]. The scalable CV error correction routines used for CV

\footnotetext{
*Electronic address: suxl@sxu.edu.cn
}

QCs were theoretically investigated [22] and experimentally demonstrated with CV multipartite entanglement of optical modes [23]. Based on off-line squeezed states and quantum nondemolition (QND) interaction between the quadrature components of two optical modes, Furusawa's group realized the QND sum gate [7] in the CV QC circuit model proposed by Filip et al. [24]. Very recently, the same group achieved an experimental demonstration of the principles of a universal one-way quantum quadratic phase gate over CVs, in which a two-mode cluster state of light is involved [8]. In contrast to the generation systems of DV cluster states of single-photons, $\mathrm{CV}$ cluster states of optical modes can be prepared unconditionally and quantum computations with CV cluster can be performed deterministically $[25,26]$. Following the first theoretical proposal on universal QC with CV cluster states [27], various protocols of cluster CV QCs for experimental implementation are studied in detail [28-30]. In experiments, CV cluster states involving four optical modes were successfully prepared [31, 32].

It was pointed out that the single-qubit and two-qubit gates used in teleportation are sufficient to construct even the most complex QCs [33]. Generally, in QCs quantum information is propagated via teleportation networks, thus quantum teleporters are key elements of building QCs [34]. By the end of the last century quantum teleportation was been experimentally realized with both DV [35-41] and CV [42-44] protocols. Later, the teleportation networks were achieved with single-photon multiparticle polarization-entanglement [45] and multipartite quadrature-entanglement of optical modes [46], respectively. These successful experiments on teleportation provide the fundamental technology to construct QCs.

In this article, we present an experimental study toward demonstrating the controlled- $X$ operation, which is an ana$\log$ of a two-qubit contolled-NOT gate in the CV regime [27]. This controlled- $X$ operation (it is also called the sum gate) based on utilizing CV four-partite cluster states of optical modes is different from that achieved in Refs. [7] and [8]. The QND sum gate in Ref. [7] belongs to the typical circuit model and the quadratic phase gate in Ref. [8] only achieves 
a single-mode operation involving a two-mode cluster state. According to our previous theoretical design (see the Sec. V in Ref. [29]) we experimentally explored the scheme of implementing the one-way controlled-X operation, a two-mode gate using the linear four-partite cluster state of optical modes, homodyne detectors and electronic feeding forward. In the operation system two CV quantum teleportation elements are included, which are used for teleporting the information of the input target and control states to the output states. The experimental results show that the amplitude quadrature of the output target is displaced a certain amount set by the input control state according to the requirement of the controlled-X operation. The measured variances of the quadratures of the output states in the case exploiting cluster quantum resources are about $1.8 \mathrm{~dB}$ below the shot noise limit (SNL) determined by the vacuum noise levels of coherent states without the existence of cluster entanglement. Based on the nonlocal and deterministic entanglement feature of $\mathrm{CV}$ cluster states the presented controlled-X operation can be also implemented nonlocally and deterministically. The construction of the experimental system for the controlled-X operation exhibits the key role of quantum teleportation in QCs, obviously. The fidelities of the output target and control states are calculated, respectively, and both of them surpass their classical limit. Since the squeezing level of the resources of the cluster state is not high enough (only $\sim 3 \mathrm{~dB}$ ) the entanglement between output target and control modes, which is necessary for further quantum information processing, was not observed in the present experiment. However, we provide a scalable experimental system and scheme toward achieving the controlled-X operation.

\section{OPERATION PRINCIPLE}

The Hamiltonian of the controlled-X operation can be written as $\hat{H}=-\hat{X}_{c} \hat{Y}_{t}$, where $\hat{X}=\hat{a}+\hat{a}^{+}$and $\hat{Y}=\left(\hat{a}-\hat{a}^{+}\right) / i$ are the amplitude and phase quadratures of an input optical mode $\hat{a}$, and the subscripts $c$ and $t$ denote the control and target modes, respectively. The ideal input-output relation of the controlled-X operation are given by [19],

$$
\begin{aligned}
& \hat{X}_{c}^{\text {out }}=\hat{X}_{c}, \quad \hat{X}_{t}^{\text {out }}=\hat{X}_{t}-\hat{X}_{c}, \\
& \hat{Y}_{c}^{\text {out }}=\hat{Y}_{c}+\hat{Y}_{t}, \quad \hat{Y}_{t}^{\text {out }}=\hat{Y}_{t} .
\end{aligned}
$$

Through the controlled-X operation the input control (target) variable $\hat{X}_{c}\left(\hat{Y}_{t}\right)$ is added to the output target (control) variable $\hat{X}_{t}^{\text {out }}\left(\hat{Y}_{c}^{\text {out }}\right)$, while $\hat{X}_{c}\left(\hat{Y}_{t}\right)$ remains unchanged. The operation results in a phase-space displacement on the amplitude quadrature of the target by an amount determined by the position (amplitude) eigenvalue $\hat{X}_{c}$ of the control and possibly establishes the quantum entanglement between the output target and control modes, which is just the aim of the controlled-X gate.

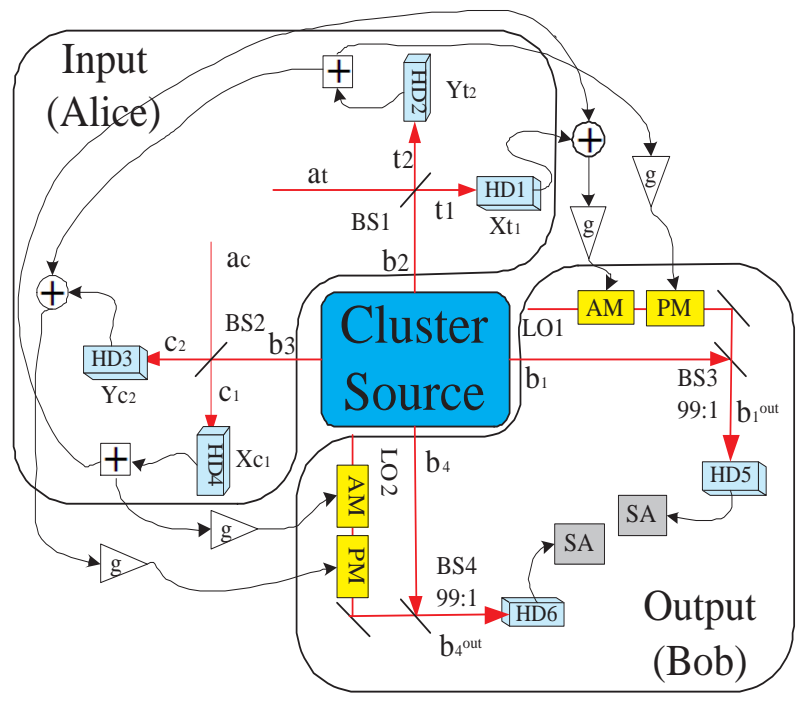

FIG. 1: (Color online) Schematic of controlled-X operation. b1-4: four submodes of CV linear four-partite cluster state from the cluster source; HD1-6: homodyne detection system; AM: amplitude modulator; PM: phase modulator; BS1 and 2: 50\% beamsplitter; BS3 and 4: 99:1 beamsplitter; at: input target mode; ac: input control mode; $\mathrm{c} 1$ and 2: the output field from BS2; 11 and 2: the output field from BS1; g: gain of feed forward circuit; $\boxplus$ : power splitter; $\oplus$ : positive power combiner; LO: auxiliary local oscillation beam; and SA: spectrum analyzer.

\section{EXPERIMENTAL METHOD}

Figure 1 shows the schematic of the controlled-X operation with the four-partite linear CV cluster state of the optical field. The four submodes $\hat{b}_{1}-\hat{b}_{4}$ of the cluster state are generated by linearly combining four quadrature-squeezed states of light produced from a pair of nondegenerate optical parametric amplifiers (NOPAs) operating at deamplification and below the oscillation threshold [31]. Compared to our previous cluster generation system [31] only a slight change is made, that is, a $1: 1$ beamsplitter (BS1) in the old system is replaced by a $4: 1$ beamsplitter thus eliminating the effect of the antisqueezing component on the squeezing component to the largest extent [29, 32]. The squeezed correlation variances of the cluster state are expressed by [29]

$$
\begin{aligned}
\hat{Y}_{b_{1}}-\hat{Y}_{b_{2}} & =\sqrt{2} e^{-r} \hat{Y}_{a_{1}}^{(0)} \\
\hat{X}_{b_{1}}+\hat{X}_{b_{2}}+\hat{X}_{b_{3}} & =\frac{\sqrt{10}}{2} e^{-r} \hat{X}_{a_{2}}^{(0)}-\frac{\sqrt{2}}{2} e^{-r} \hat{Y}_{a_{4}}^{(0)} \\
-\hat{Y}_{b_{2}}+\hat{Y}_{b_{3}}+\hat{Y}_{b_{4}} & =-\frac{\sqrt{10}}{2} e^{-r} \hat{X}_{a_{3}}^{(0)}+\frac{\sqrt{2}}{2} e^{-r} \hat{Y}_{a_{1}}^{(0)} \\
\hat{X}_{b_{3}}-\hat{X}_{b_{4}} & =-\sqrt{2} e^{-r} \hat{Y}_{a_{4}}^{(0)}
\end{aligned}
$$

where $r$ stands for the squeezing parameter of the squeezed states, which depends on the strength and the time of parametric interaction in NOPA. We assumed that $r$ of the four squeezed states is identical, which is not difficult to reach experimentally by balancing the configuration and system pa- 
rameter of NOPAs. The values of $r$ are between zero and positive infinite with $r=0$ for no squeezing and $r \rightarrow \infty$ for the ideal squeezing. However, the ideal squeezing limit can not be achieved in experiments since it requires infinite energy. $\hat{X}_{a i}^{(0)}$ and $\hat{Y}_{a i}^{(0)}(i=1-4)$ denote the quadrature amplitudes and phases of seed optical beams initially injected into NOPAs, respectively [29, 31]. The variances of the seed beams in coherent states are normalized, that is $V\left(\hat{X}_{a i}^{(0)}\right)=V\left(\hat{Y}_{a i}^{(0)}\right)=1$. The submodes $\hat{b}_{2}, \hat{b}_{3}$ and $\hat{b}_{1}, \hat{b}_{4}$ are distributed to input (Alice) and output (Bob) of the controlled-X gate, respectively.

At Alice, submodes $b_{2}$ and $b_{3}$ are mixed with the $\operatorname{target}\left(\hat{a}_{t}\right)$ and the control $\left(\hat{a}_{c}\right)$ signals on $1: 1$ beamsplitters BS1 and BS2, respectively. The amplitude and phase quadratures of the output target (control) modes $\hat{t}_{1}$ and $\hat{t}_{2}\left(\hat{c}_{1}\right.$ and $\left.\hat{c}_{2}\right)$ from BS1 (BS2) are detected by a pair of balanced homodyne detectors HD1 and HD2 (HD3 and HD4) actively locked to be 0 and $90^{\circ}$ out of phase, respectively. The measured conjugate quadratures of optical modes are denoted by $\hat{X}_{t 1}, \hat{Y}_{t 2}$ and $\hat{X}_{c 1}, \hat{Y}_{c 2}$, respectively, for the target and the control. The measured photocurrents of $\hat{X}_{c 1}$ and $\hat{Y}_{t 2}$ are split by two power splitters $(\boxplus)$, respectively. Then a half of the photocurrent $\hat{X}_{c 1}$ $\left(\hat{Y}_{t 2}\right)$ is added to $\hat{X}_{t 1}\left(\hat{Y}_{c 2}\right)$ by a positive power combiner $(\oplus)$. The photocurrents $\left(\hat{X}_{t 1}+\hat{X}_{c 1}\right)$ and $\left(\hat{Y}_{t 2}+\hat{Y}_{c 2}\right)$, as well as the remaining other half of $\hat{X}_{c 1}$ and $\hat{Y}_{t 2}$ are sent to Bob through four classical channels with a suitable electronic gain $g(\triangleleft)$, where Bob uses them to impose amplitude and phase modulations on two bright laser beams in coherent states (LO1 and LO2) by means of amplitude (AM) and phase (PM) modulators, respectively. By mixing the modulated LO1 (LO2) with the submode $\hat{b}_{1}\left(\hat{b}_{4}\right)$ remaining by Bob on a $99: 1$ highly reflective beamsplitter BS3 (BS4), a displacement in the phasespace proportional to the amplitude and phase modulations is imposed on $\hat{b}_{1}\left(\hat{b}_{4}\right)$. Because of cluster entanglement among the four submodes $\hat{b}_{1}-\hat{b}_{4}$, Alice's two Bell-state detections collapse both submodes $\hat{b}_{1}$ and $\hat{b}_{4}$ into a state conditioned on the measurement outcomes $\hat{X}_{t 1}, \hat{Y}_{t 2}$ and $\hat{X}_{c 1}, \hat{Y}_{c 2}$. The amplitude quadrature $\hat{X}_{t}^{\text {out }}\left(\hat{X}_{c}^{\text {out }}\right)$ and the phase quadrature $\hat{Y}_{t}^{\text {out }}$ $\left(\hat{Y}_{r}^{\text {out }}\right)$ of the displaced outcome $\hat{b}_{1}^{\text {out }}\left(\hat{b}_{4}^{\text {out }}\right)$ are expressed by [29]

$$
\begin{aligned}
\hat{X}_{t}^{\text {out }} & =\hat{X}_{b_{1}}+\sqrt{2} g \hat{X}_{t_{1}}+\sqrt{2} g \hat{X}_{c_{1}} \\
& =\sqrt{\frac{5}{2}} e^{-r} \hat{X}_{a_{2}}^{(0)}-\sqrt{\frac{1}{2}} e^{-r} \hat{Y}_{a_{4}}^{(0)}+\hat{X}_{t}-\hat{X}_{c} \\
\hat{Y}_{t}^{\text {out }} & =\hat{Y}_{b_{1}}-\sqrt{2} g \hat{Y}_{t_{2}} \\
& =\sqrt{2} e^{-r} \hat{Y}_{a_{1}}^{(0)}+\hat{Y}_{t} \\
\hat{X}_{c}^{\text {out }} & =\hat{X}_{b_{4}}-\sqrt{2} g \hat{X}_{c_{1}} \\
& =\sqrt{2} e^{-r} \hat{Y}_{a_{4}}^{(0)}+\hat{X}_{c} \\
\hat{Y}_{c}^{\text {out }} & =\hat{Y}_{b_{4}}-\sqrt{2} g \hat{Y}_{t_{2}}+\sqrt{2} g \hat{Y}_{c_{2}} \\
& =-\sqrt{\frac{5}{2}} e^{-r} \hat{X}_{a_{3}}^{(0)}+\sqrt{\frac{1}{2}} e^{-r} \hat{Y}_{a_{1}}^{(0)}+\hat{Y}_{t}+\hat{Y}_{c} .
\end{aligned}
$$

In cluster QC language the displacement operation is equivalent to feed-forward the measurement results of the submodes $\hat{b}_{2}$ and $\hat{b}_{3}$ on the remained submodes $\hat{b}_{1}$ and $\hat{b}_{4}$. In the experiment, the gain $g$ of all feed-forward circuits is carefully adjusted to 1 , which corresponds to realizing a controlled-X operation in Eq.(1). It is obvious that from Eqs. (6) - (9) that in the limit of infinite squeezing $(r \rightarrow \infty)$, the amplitude quadrature of the output target, $\hat{X}_{t}^{\text {out }}$, was displaced an amount $\hat{X}_{c}$ determined by the amplitude of the input control field. It means that the ideal controlled-X operation was completed. For practical experiments with a finite value of $r$, some noise resulting from imperfect squeezing will be added on the $\hat{X}_{t}^{\text {out }}$, thus the fidelity of the outcome state will reduce. However, the fidelity using finite cluster entanglement of $r \neq 0$ will be higher than its classical limit which is measured in the case without the existence of cluster state. To verify the performance of the controlled-X operation the outcome values of $\hat{X}_{t}^{\text {out }}, \hat{Y}_{t}^{\text {out }}$ and $\hat{X}_{c}^{\text {out }}, \hat{Y}_{c}^{\text {out }}$ are detected by the homodyne detectors, HD5 and HD6, respectively.

The cluster source comprises a master-pump laser, a pair of NOPAs and some linearly optical elements, which are not shown in Fig. 1 (see Ref. [31] for details). A continuous wave intracavity frequency-doubled and frequency-stabilized Nd:YAP/KTP (Nd-doped YAlO3 perovskite / potassium titanyl phosphate) laser (made by Yuguang Co. Ltd., F-VIB) [47, 48] serves as the master laser of the experimental system. The output second-harmonic wave at $540 \mathrm{~nm}$ is used for the pump laser of the two NOPAs to produce four two-mode squeezed states at $1080 \mathrm{~nm}$ via intracavity frequency downconversion. The four squeezed states are transformed to a linear four-partite cluster state with optical beamsplitters [31]. In the system, all the optical beams at coherent states, including the target signal $\left(\hat{a}_{t}\right)$, the control signal $\left(\hat{a}_{c}\right), \mathrm{LO} 1, \mathrm{LO} 2$, and the local oscillation beams used in homodyne detectors (HD1-6), originate from the subharmonic output of the master laser at $1080 \mathrm{~nm}$. During the experiment the pump power of the NOPAs are kept at $175 \mathrm{~mW}$ which is below the oscillation threshold of $230 \mathrm{~mW}$, and the intensity of the signals into NOPAs at $1080 \mathrm{~nm}$ is $10 \mathrm{~mW}$. When NOPAs operate at deamplification (the pump light and the injected signals are $\pi$ out of phase), the intensity of each submode of the obtained cluster state is about $30 \mu \mathrm{W}$. The power of the local oscillation beam in each HD is about $4 \mathrm{~mW}$ and the intensity of LO1 (LO2) is $54 \mu \mathrm{W}$. All the squeezed correlation variances of the cluster state measured under the previous conditions are about $3 \mathrm{~dB}$ below the corresponding SNL (the equivalent squeezing parameter $r$ equals to 0.35 ). The electronic gain $g$ in classical channels is carefully adjusted to $1.00 \pm 0.05$ according to the method described in Ref. [44].

\section{EXPERIMENTAL RESULTS}

To quantify the performance of the controlled-X operation, the noise variances of $\hat{X}_{t(c)}^{\text {out }}$ and $\hat{Y}_{t(c)}^{\text {out }}$ in Eqs. (6) - (9) are measured by HD5(6) and are recorded by a following spectrum analyzer (SA) with the resolution bandwidth of $30 \mathrm{kHz}$ and video bandwidth of $100 \mathrm{~Hz}$. The measured noise powers of $\hat{X}_{t(c)}^{\text {out }}$ and $\hat{Y}_{t(c)}^{\text {out }}$ with the vacuum input $\left(\hat{a}_{t}\right.$ and $\hat{a}_{c}$ are vacuum state) are shown in Fig. 2. The noise powers of the 


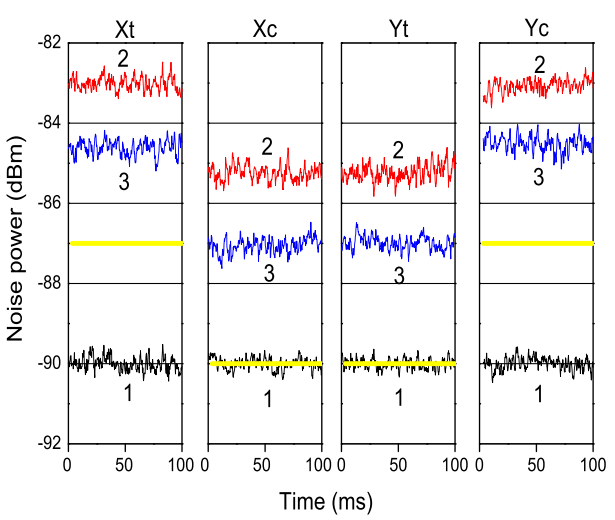

FIG. 2: (Color online) Noise power of the quadratures with vacuum inputs. Black (1) lines: noise of vacuum state; red (2) lines: noise variances of output quadratures without cluster state; blue (3) lines: noise variances of output quadratures with cluster state; and yellow (straight) lines: noise variances of output quadratures in the ideal case.

two input vacuum states serve as the SNL [(black) 1 lines]. In case of infinite squeezing [(yellow) straight lines], the noise variances of $\hat{X}_{t}^{\text {out }}$ and $\hat{Y}_{c}^{\text {out }}$ are $3 \mathrm{~dB}$ above the SNL due to the effect of $\hat{X}_{c}\left(\hat{Y}_{t}\right)$, while noise variances of $\hat{X}_{c}^{\text {out }}$ and $\hat{Y}_{t}^{\text {out }}$ remain at SNL [see Eq. (1)]. The (red) 2 lines and the (blue) 3 lines illustrate the performance of the controlled-X operation without and with using the quantum entanglement of the cluster state, respectively. The (red) 2 lines are measured by replacing each cluster submode with a coherent light of identical intensity. The measured values of $\hat{X}_{t}^{\text {out }}, \hat{X}_{c}^{\text {out }}, \hat{Y}_{t}^{\text {out }}$, and $\hat{Y}_{c}^{\text {out }}$ are $6.95,4.76,4.77$ and $6.93 \mathrm{~dB}$ above the SNL, respectively. The variances of $\hat{X}_{t}^{\text {out }}, \hat{X}_{c}^{\text {out }}, \hat{Y}_{t}^{\text {out }}$, and $\hat{Y}_{c}^{\text {out }}$ measured with the existence of the cluster state [(blue) 3 lines] are 5.39, 2.95, 3.01, $5.50 \mathrm{~dB}$ above the SNL, respectively. The (blue) 3 lines with the cluster state are $\sim 1.8 \mathrm{~dB}$ below that without using the cluster [(red) 2 lines]. It means that the precision of the controlled-X operator increases about $1.8 \mathrm{~dB}$ with respect to its classical copies. We use the fidelity formula $F=\left\{\operatorname{Tr}\left[\left(\sqrt{\hat{\rho}_{1}} \hat{\rho}_{2} \sqrt{\hat{\rho}_{1}}\right)^{1 / 2}\right]\right\}^{2}$, which denotes the overlap between the experimental obtained output state $\hat{\rho}_{2}$ and ideal output sate $\hat{\rho}_{1}$, to quantify the performance of controlled-X operation. The fidelity for two Gaussian states $\hat{\rho}_{1}$ and $\hat{\rho}_{2}$ with covariance matrices $\mathbf{A}_{i}$ and mean amplitudes $\alpha_{i} \equiv\left(\alpha_{i X}, \alpha_{i Y}\right)$ $(i=1,2)$ is expressed by [49, 50]

$$
F=\frac{2}{\sqrt{\Delta+\delta}-\sqrt{\delta}} \exp \left[-\beta^{T}\left(\mathbf{A}_{1}+\mathbf{A}_{2}\right)^{-1} \beta\right]
$$

where $\Delta=\operatorname{det}\left(\mathbf{A}_{1}+\mathbf{A}_{2}\right), \delta=\left(\operatorname{det} \mathbf{A}_{1}-1\right)\left(\operatorname{det} \mathbf{A}_{2}-1\right)$, $\beta=\alpha_{2}-\alpha_{1}, \mathbf{A}_{1}$ and $\mathbf{A}_{2}$ for the ideal $\left(\hat{\rho}_{1}\right)$ and experimental $\left(\hat{\rho}_{2}\right)$ output states, respectively. For our case, the covariance matrices $\mathbf{A}_{i}(i=1,2)$ for the target mode are given by
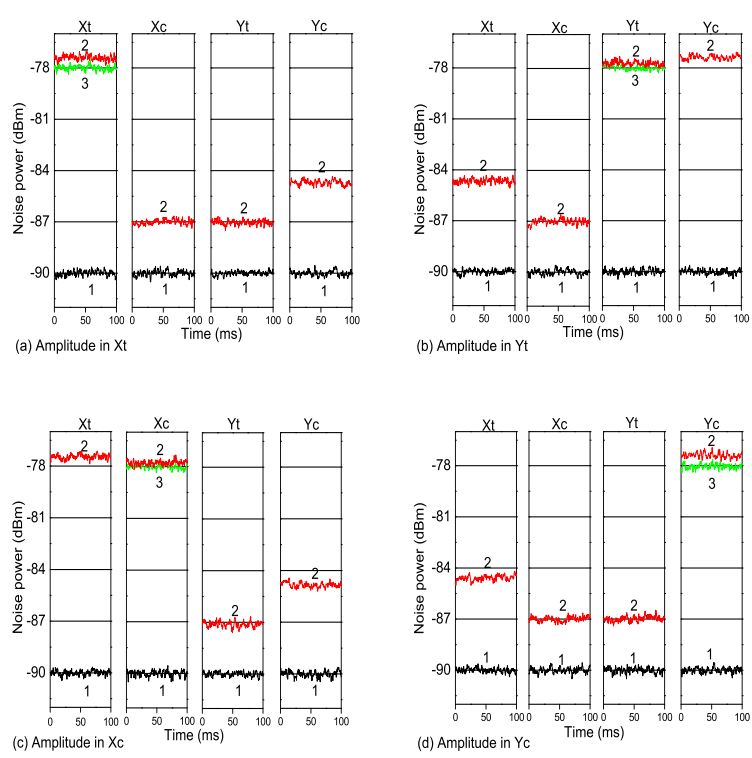

FIG. 3: (Color online) Noise power of the quadratures with four different coherent input quadratures. Black (1) lines: noises of vacuum state; red (2) lines: output variances with cluster state; and green (3) lines: input variances.

$$
\begin{aligned}
& \mathbf{A}_{1}=\left[\begin{array}{cc}
V\left(X_{t}-X_{c}\right) & 0 \\
0 & V\left(Y_{t}\right)
\end{array}\right]=\left[\begin{array}{ll}
2 & 0 \\
0 & 1
\end{array}\right], \\
& \mathbf{A}_{2}=\left[\begin{array}{cc}
V\left(X_{t}^{\text {out }}\right) & 0 \\
0 & V\left(Y_{t}^{\text {out }}\right)
\end{array}\right]
\end{aligned}
$$

Similarly, we can write out the covariance matrices for the control mode. In case of infinite squeezing, both fidelities for the control and target states $F_{c}$ and $F_{t}$ equal 1 , which can be calculated from Eqs. (6) $-(9)$ with $r \rightarrow \infty$. Without using cluster resources $(r=0)$, the fidelity for both $\hat{a}_{t}$ and $\hat{a}_{c}$ states is 0.73 . When the cluster states are used, the obtained fidelities of $\hat{a}_{t}$ and $\hat{a}_{c}$ are both 0.87 , which are about 0.14 better than those without using entanglement.

To simulate the performance of the controlled-X gate under the coherent input signals with nonzero average intensity, we modulate the input $\hat{a}_{t}$ and $\hat{a}_{c}$ optical beams with amplitude and phase modulators at $2 \mathrm{MHz}$, (not shown in Fig. 1). The coherent excitations of four different quadratures corresponding to four input states with individual modulation are investigated. The traces in Figs. 3 (a), (b), (c) and (d) correspond to four different input states: (a) and (b) for the amplitude and phase modulation of the input target $a_{t}$ and (c) and (d) for the amplitude and phase modulation of the input control $a_{c}$, respectively. In the four different modulation models, the intensity of the imposed modulation signals is identical. The (black) 1 lines and the (green) 3 lines in Fig. 3 correspond to the SNL and the quadratures of the input signals, respectively. The quadratures of the outcome fields are shown with the (red) 2 lines. All coherent amplitudes of the input 
quadratures are $12 \mathrm{~dB}$ above the SNL due to the same modulation strength, which are measured by HD5 and HD6 in the case blocking the cluster beams. We can see from Figs. 3 (b) and (c) that the quadrature $\hat{Y}_{t}\left(\hat{X}_{c}\right)$ of the input state is coupled into $\hat{Y}_{c}^{\text {out }}\left(\hat{X}_{t}^{\text {out }}\right)$ of the outcome state, while $\hat{Y}_{c}=\hat{Y}_{c}^{\text {out }}$ $\left(\hat{X}_{t}=\hat{X}_{t}^{\text {out }}\right)$ is preserved. Figs. 3 (a) and (d) show that the quadratures $\hat{X}_{t}$ and $\hat{Y}_{c}$ of input states are not coupled to any output quadratures, in which, however, the variances of the outcome quadratures are a little higher than that of the input states due to the effect of imperfect squeezing.

\section{CONCLUSION}

In conclusion, an experimental study toward demonstrating the CV controlled-X logical operation based on four-partite cluster states of electro-magnetic fields is presented. In the experiment the information encoded in the input target and control states are teleported to the outcome states via quantum channels depending on multipartite cluster entanglement among quadrature components of optical modes and electronic classical channels. The one-way controlled-X gate can be regarded as to be constructed by two $\mathrm{CV}$ quantum tele- porters based on CV quantum entanglement among modes $\hat{b}_{1}, \hat{b}_{2}, \hat{b}_{3}$ and $\hat{b}_{4}, \hat{b}_{2}, \hat{b}_{3}$, respectively. The difference between these teleporters and the normal teleportation systems [42-44] is that there are two input states (target and control) in these teleporters and both of their information is transmitted to the two output states simultaneously. To achieve the basic operation of one-way quantum computation the four-partite cluster entanglement plays irreplaceable roles. In CV QCs, we have to establish entanglement between the output target and control modes for further quantum information processing. According to the theoretical calculation [51] for achieving the entanglement of the two output states the initial squeezing degree of cluster resources should be higher than $\sim 7 \mathrm{~dB}$. Although the entanglement between output states was not observed in the present experiment due to the absence of better cluster resources, the proof-of-principle experiment proves that the controlled-X operation of one-way QC can be unconditionally demonstrated with the designed system if CV cluster states of higher entanglement are available.

This research was supported by the NSFC (Grant Nos. 60736040 and 10804065), NSFC Project for Excellent Research Team (Grant No. 60821004), and the National Basic Research Program of China (Grant No. 2007BAQ03918).
[1] C. Monroe, D. M. Meekhof, B. E. King, W. M. Itano, and D. J. Wineland, Phys. Rev. Lett. 75, 4714 (1995).

[2] T. B. Pittman, B. C. Jacobs, and J. D. Franson, Phys. Rev. Lett. 88, 257902 (2002).

[3] F. Schmidt-Kaler, H. Häffner, M. Riebe, S. Gulde, G. P. T. Lancaster, T. Deuschle, C. Becher, C. F. Roos, J. Eschner, and R. Blatt, Nature, 422, 408, (2003).

[4] D. Leibfried, B. DeMarco, V. Meyer, D. Lucas, M. Barrett, J. Britton, W. M. Itano, B. Jelenkovic, C. Langer, T. Rosenband, and D. J. Wineland, Nature, 422, 412, (2003).

[5] X. Li, Y. Wu, D. Steel, D. Gammon, T. H. Stievater, D. S. Katzer, D. Park, C. Piermarocchi, and L. J. Sham, Science, 301, 809, (2003).

[6] Z. Zhao, A. N. Zhang, Y. A. Chen, H. Zhang, J. F. Du, T. Yang, and J. W. Pan, Phys. Rev. Lett. 94, 030501 (2005).

[7] J. I. Yoshikawa, Y. Miwa, A. Huck, U. L. Andersen, P. van Loock, and A. Furusawa, Phys. Rev. Lett. 101, 250501 (2008).

[8] Y. Miwa, J. I. Yoshikawa, P. van Loock, and A. Furusawa, Phys. Rev. A 80, 050303(R), (2009).

[9] M. A. Nielsen and I. L. Chuang, Quantum computation and Quantum information (Cambridge University Press, Cambridge, 2000).

[10] I. L. Chuang, L. M. K. Vandersypen, X. Zhou, D. W. Leung, S. Lloyd, Nature, 393, 143, (1998).

[11] D. Gottesman, and I. L. Chuang, Nature, 402, 390, (1999).

[12] S. Gulde, M. Riebe, G. P. T. Lancaster, C. Becher, J. Eschner, H. Häffner, F. Schmidt-Kaler, I. L. Chuang, and R. Blatt, Nature, 421, 48, (2003).

[13] C. Y. Lu, D. E. Browne, T. Yang, and J. W. Pan, Phys. Rev. Lett. 99, 250504 (2007).

[14] A. Politi, J. C. F. Matthews, J. L. O’Brien, Science, 325, 1221 (2009).

[15] R. Raussendorf and H. J. Briegel, Phys. Rev. Lett. 86, 5188 (2001).
[16] P. Walther, K. J. Resch, T. Rudolph, E. Schenck, H. Weinfurter, V. Vedral, M. Aspelmeyer, and A. Zeilinger, Nature, 434, 169 (2005).

[17] R. Prevedel, P. Walther, F. Tiefenbacher, P. Böhi, R. Kaltenbaek, T. Jennewein and A. Zeilinger, Nature, 445, 65 (2007).

[18] K. Chen, C. M. Li, Q. Zhang, Y. A. Chen, A. Goebel, S. Chen, A. Mair, and J. W. Pan, Phys. Rev. Lett. 99, 120503 (2007).

[19] S. Lloyd and S. L. Braunstein, Phys. Rev. Lett. 82, 1784 (1999).

[20] T. C. Ralph, A. Gilchrist, G. J. Milburn, W. J. Munro, and S. Glancy, Phys. Rev. A 68, 042319 (2003).

[21] D. Gottesman, A. Kitaev, and J. Preskill, Phys. Rev. A 64, 012310 (2001).

[22] S. L. Braunstein, Phys. Rev. Lett. 80, 4084 (1998).

[23] T. Aoki, G. Takahashi, T. Kajiya, J. Yoshikawa, S. L. Braunstein, P. van Loock, A. Furusawa, Nature Physics 5, 541 (2009).

[24] R. Filip, P. Marek, and U. L. Andersen, Phys. Rev. A 71, 042308 (2005).

[25] J. Zhang and S. L. Braunstein, Phys. Rev. A 73, 032318 (2006).

[26] P. van Loock, C. Weedbrook, and M. Gu, Phys. Rev. A 76, 032321 (2007).

[27] N. C. Menicucci, P. van Loock, M. Gu, C. Weedbrook, T. C. Ralph, and M. A. Nielsen, Phys. Rev. Lett. 97, 110501 (2006).

[28] P. van Loock, J. Opt. Soc. Am. B 24, 340 (2007).

[29] A. H. Tan, C. D. Xie, and K. C. Peng, Phys. Rev. A 79, 042338 (2009).

[30] M. Gu, C. Weedbrook, N. C. Menicucci, T. C. Ralph, and P. van Loock, Phys. Rev. A 79, 062318 (2009).

[31] X. L. Su, A. H. Tan, X. J. Jia, J. Zhang, C. D. Xie, and K. C. Peng, Phys. Rev. Lett. 98, 070502 (2007).

[32] M. Yukawa, R. Ukai, P. van Loock, and A. Furusawa, Phys. Rev. A 78, 012301 (2008).

[33] S. Lloyd, Phys. Let. A 167, 255 (1992).

[34] P. Hemmer and J. Wrachtrup, Science, 324, 473 (2009).

[35] D. Bouwmeester, J. W. Pan, K. Mattle, M. Eibl, H. Weinfurter, 
and A. Zeilinger, Nature, 390, 575 (1997).

[36] D. Boschi, S. Branca, F. De Martini, L. Hardy, and S. Popescu, Phys. Rev. Lett., 80, 1121 (1998).

[37] M. Riebe, H. Häffner, C. F. Roos, W. Hänsel, J. Benhelm, G. P. T. Lancaster, T. W. Körber, C. Becher, F. Schmidt-Kaler, D. F. V. James, and R. Blatt, Nature, 429, 734 (2004).

[38] M. D. Barrett, J. Chiaverini, T. Schaetz, J. Britton, W. M. Itano, J. D. Jost, E. Knill, C. Langer, D. Leibfried, R. Ozeri, and D. J. Wineland, Nature, 429, 737 (2004).

[39] Y. F. Huang, X. F. Ren, Y. S. Zhang, L. M. Duan, and G. C. Guo, Phys. Rev. Lett, 93, 240501 (2004).

[40] J. F. Sherson, H. Krauter, R. K. Olsson, B. Julsgaard, K. Hammerer, I. Cirac, and E. S. Polzik, Nature, 443, 557 (2006).

[41] S. Olmschenk, D. N. Matsukevich, P. Maunz, D. Hayes, L.-M. Duan, and C. Monroe, Science, 323, 486 (2009).

[42] A. Furusawa, J. L. Sorenson, S. L. Braunstein, C. A. Fuchs, H. J. Kimble, E. S. Polzik, Science, 282, 706 (1998).

[43] W. P. Bowen, N. Treps, B. C. Buchler, R. Schnabel, T. C. Ralph,
Hans-A. Bachor, T. Symul, and P. K. Lam, Phys. Rev. A 67, 032302 (2003).

[44] T. C. Zhang, K. W. Goh, C. W. Chou, P. Lodahl, and H. J. Kimble, Phys. Rev. A, 67, 033802 (2003).

[45] Z. Zhao, Y. A. Chen, A. N. Zhang, T. Yang, H. J. Briegel, and J. W. Pan, Nature, 430, 54 (2004).

[46] H. Yonezawa, T. Aoki, and A. Furusawa, Nature, 431, 430 (2004).

[47] X. Y. Li, Q. Pan, J. T. Jing, J. Zhang, C. D. Xie, and K. C. Peng, Phys. Rev. Lett. 88, 047904 (2002).

[48] X. J. Jia, X. L. Su, Q. Pan, J. R. Gao, C. D. Xie, and K. C. Peng, Phys. Rev. Lett. 93, 250503 (2004).

[49] H. Nha, and H. J. Carmichael, Phys. Rev. A, 71, 032336 (2005).

[50] H. Scutaru, J. Phys. A 31, 3659 (1998).

[51] Lu-Ming Duan, G. Giedke, J. I. Cirac, and P. Zoller, Phys. Rev. Lett. 84, 2722 (2000). 